Philipp Müller ${ }^{1}$

Johannes Gutenberg Universität Mainz

\title{
Jugend und Kirche in Deutschland - Eine pastoraltheologische Skizze
}

Im Jahr 1983 hat Klaus Hemmerle (1929-1994), der Bischof von Aachen und vormalige Freiburger Professor für Religionsphilosophie, in der „Internationalen katholischen Zeitschrift »Communio«" einen wegweisenden Artikel zum Thema "Jugendpastoral" verfasst. Der Titel des Aufsatzes besteht aus zwei Fragen, die auch heute, nach mehr als 30 Jahren, nichts an Aktualität eingebüßt haben. Er lautet: „Was fängt die Jugend mit der Kirche an? Was fängt die Kirche mit der Jugend an?“" Eine ähnliche Perspektive nimmt auch dieser Beitrag ein. Er sucht zunächst zu ergründen, was heutige Jugendliche in Deutschland über die Kirche den-

1 Philipp Müller, Prof. Dr. theol. Studium der Kath. Theologie und Geschichte, Priesterweihe 1991, Regens des Priesterseminars der Erzdiözese Freiburg, Habilitation für Pastoraltheologie und Homiletik an der Kath.-Theol. Fakultät der Universität Freiburg i. Br. Seit 2011 Professor für Pastoraltheologie an der Johannes Gutenberg-Universität in Mainz. Berater der Deutschen Bischofskonferenz in der Kommission für „Geistliche Berufe und Kirchliche Dienste“. Forschungsschwerpunkte (u.a.): Seelsorgelehre, Martyria als pastoraler Grundvollzug, Geschichte der Pastoral und der Pastoraltheologie, Christliche Spiritualität in heutiger Zeit. E-mail: ph.mueller@uni-mainz.de.

2 K. Hemmerle, Was fängt die Jugend mit der Kirche an? Was fängt die Kirche mit der Jugend an?, „Internationale Katholische Zeitschrift Communio“ Nr. 12 (1983), S. 306-317. Hier zitiert nach ders.: Spielräume Gottes und der Menschen. Beiträge zu Ansatz und Feldern kirchlichen Handeln, [in:] Ausgewählte Schriften (Bd. 4), Freiburg-Basel-Wien 1996, S. 324-339. 


\section{Philipp Müller}

ken. In einem zweiten Schritt erfolgt ein Perspektivenwechsel, wenn es um die konkrete Praxis und theoretische Konzepte der Jugendpastoral in Deutschland geht. Zum Abschluss wird ein Diktum von Klaus Hemmerle über die Jugendpastoral, das ein Leitwort für jedwede Seelsorge mit ganz unterschiedlichen Menschen sein kann, aufgegriffen und in seinen Konsequenzen bedacht.

\section{Wie sieht die heutige Jugend die Kirche?}

Deutschland hat gegenwärtig etwas mehr als 80 Millionen Einwohner. Ein Blick auf die Bevölkerungspyramide weist Deutschland als ein überaltertes Land mit zu wenig Kindern und Jugendlichen aus - ist Deutschland doch schon länger ein Land mit einer der niedrigsten Geburtenquoten überhaupt. ${ }^{3}$ Von den 80 Millionen Einwohnern sind knapp 30\% katholisch; fast ebenso viele gehören im Ursprungsland der Reformation einer anderen christlichen Konfession an. Weil die Taufe gesellschaftlich gesehen nach wie vor eine hohe Akzeptanz besitzt und sie meistens im ersten Lebensjahr gespendet wird, ${ }^{4}$ sind vermutlich gut die Hälfte der Jugendlichen getauft. Dabei ist das konfessionelle Gewicht regional unterschiedlich verteilt: Zum Beispiel sind im Saarland (in der Nähe zu Frankreich), im Rheinland oder in Teilen Bayerns deutlich mehr als die Hälfte der Jugendlichen katholisch, während im Norden Deutschlands viele evangelischen Glaubens sind und im Osten Deutschlands der Großteil der Menschen keiner christlichen Konfession angehört.

So gut wie alle katholisch getauften Kinder gehen mit etwa 9 Jahren zur Erstkommunion; sie bereiten sich mehrere Monate auf dieses Fest vor. Vier bis sechs Jahre später sind sie als Jugendliche eingeladen, das Firmsakrament zu empfangen; knapp sieben von zehn Jugendlichen neh-

3 Zur demographischen Entwicklung und ihren Folgen vgl. A. Rödder, 21.0. Eine kurze Geschichte der Gegenwart, 2. Aufl., München 2015, S. 173-183.

${ }_{4}$ Vgl. Katholische Kirche in Deutschland: Zahlen und Fakten 2014/15, Hg. Sekretariat der Deutschen Bischofskonferenz (Arbeitshilfen 275), S. 36. Zur Situation der Kirche in Deutschland vgl. auch Ph. Müller, Die katholische Kirche in Deutschland - Eine Bestandsaufnahme, „Polonia Sacra“ 20 (2016) Nr 2 (42), S. 47-69. 
men diese Einladung an und werden gefirmt. In der Vorbereitung junger Menschen auf diese beiden Sakramente leisten Priester sowie Pastoral- und Gemeindereferent(inn)en, aber auch ehrenamtliche Katechetinnen und Katecheten einen wichtigen Beitrag. Freilich befällt manche Engagierte nach der Erstkommunion oder der Firmung ein ungutes Gefühl; denn nach dem großen Fest kommen nur noch wenige Kinder und Jugendliche zum Sonntagsgottesdienst - es sei denn, jemand ist Ministrant oder gehört einer Jugendgruppe an. Diese Entwicklung ist schon länger im Gang. So hat die Pastoralkommission der Deutschen Bischofskonferenz bereits vor mehr als 20 Jahren davon gesprochen, dass die Firmung häufig „für die Heranwachsenden das »Abschiedsfest « von der Kirche " ${ }^{\text {}}$ ist.

An öffentlichen Schulen ist der Religionsunterricht in fast allen Bundesländern ordentliches Lehrfach; er ist durch das Grundgesetz der Bundesrepublik Deutschland sogar staatlich abgesichert. ${ }^{6}$ Der Religionsunterricht steht zwar unter staatlicher Aufsicht, aber die Verantwortung für die Lehrinhalte liegt bei den Kirchen; die Religionslehrer werden wiederum vom Staat bezahlt. Dabei versteht sich der schulische Religionsunterricht in Deutschland ausdrücklich nicht als Katechese, die den christlichen Glauben wecken oder vertiefen will, sondern er leitet sich vom Bildungsauftrag der Schule ab; als solcher fördert der Religionsunterricht nach eigenem Selbstverständnis die „kulturelle Kontinuität, unterstützt die Identitätsfindung der Schüler, relativiert gesellschaftliche Absolutheitsansprüche und vermittelt so Schlüsselqualifikationen für die individuelle und soziale Lebensgestaltung"7.

Wie aber stehen heutige Jugendliche zu Kirche und Glaube? Hier ist eine wissenschaftliche Studie erhellend, die auf einem internationalen Sample beruht und der qualitative und quantitative Erhebungen

5 Sakramentenpastoral im Wandel. Überlegungen zur gegenwärtigen Praxis der Feier der Sakramente - am Beispiel von Taufe, Erstkommunion und Firmung, Juli 1993, Hg. Sekretariat der Deutschen Bischofskonferenz (Die Deutschen Bischöfe - Pastoral-Kommission 12), S. 48.

6 In Artikel 7 des Grundgesetzes heißt es: „Der Religionsunterricht ist in den öffentlichen Schulen mit Ausnahme der bekenntnisfreien Schulen ordentliches Lehrfach. Unbeschadet des staatlichen Aufsichtsrechtes wird der Religionsunterricht in Übereinstimmung mit den Grundsätzen der Religionsgemeinschaft erteilt.“ (Absatz 3).

7 W. Simon, Religionsunterricht. Praktisch-theologisch, [in:] Lexikon für Theologie und Kirche, 3. Aufl. Bd. 8, S. 1077-1079, S. 1077. 
zugrunde liegen; sie berücksichtigt sowohl die Selbstwahrnehmung von Jugendlichen als auch gesellschaftliche Aspekte. ${ }^{8}$ Diese Studie nennt fünf unterschiedliche Typen, die auch auf jene Jugendlichen zutreffen, die katholisch getauft sind und den Religionsunterricht besuchen:

- Christlich-kirchliche Jugendliche: Sie fühlen sich im christlichen Glauben zuhause, nehmen überdurchschnittlich häufig an kirchlichen Vollzügen teil und bezeichnen sich selbst als gläubig.

- Christlich-autonome Jugendliche: Für sie sind christliche Religion und Moderne durchaus miteinander kompatibel. Der Glaube kann eine Orientierungshilfe für das eigene Leben sein, doch legen sie Wert darauf, ihren Glauben unabhängig von der kirchlich-institutionalisierten Form leben zu können.

- Konventionell-religiöse Jugendliche: Auch für sie ist die Religion eine Orientierungshilfe und ein Wert, ohne groß darüber zu sprechen, weil die Religion zum Leben mehr oder weniger selbstverständlich dazu gehört.

- Autonom-religiöse Jugendliche: Sie sind nicht areligiös, gehen aber davon aus, dass eine authentische Spiritualität jenseits religiöser Institutionen selbst konstruiert werden muss; dem christlichen Offenbarungsglauben stehen sie distanziert bis ablehnend gegenüber.

- Nicht-religiöse Jugendliche: Sie lehnen die Existenz Gottes und religiöse Werte dezidiert ab und trauen dem Gottesglauben keine orientierende Kraft für das Leben zu, weil sie ihn für eine Illusion halten.

Anzumerken ist, dass infolge des gesellschaftlichen Individualisierungsprozesses die Jugendlichen auch in Fragen von Glaube und Religion selbst entscheiden möchten - nicht nur die christlich-autonomen oder autonomreligiösen, bei denen dieser Zug besonders ausgeprägt ist. ${ }^{9}$ Dies hat wiede-

8 Vgl. hierzu H.-G. Ziebertz, B. Kalbeim, U. Riegel, Religiöse Signaturen heute. Ein religionspädagogischer Beitrag zur empirischen Jugendforschung, [in:] Religionspädagogik in pluraler Gesellschaft (Bd. 3), Freiburg 2003; A. Prokopf, Religiosität Jugendlicher. Eine qualitativ-empirische Untersuchung auf den Spuren korrelativer Konzeptionen, [in:] Praktische Theologie heute (Bd. 98), Stuttgart 2008, S. $92 f$.

9 Vgl. H. Hobelsberger, Jugendpastoral des Engagements. Eine praktisch-theologische Reflexion und Konzeption des sozialen Handelns Jugendlicher, [in:] Studien zur Theologie und Praxis der Seelsorge (Bd. 67), Würzburg 2006, S. 47-57. 
rum zur Konsequenz, dass die Anwendung von Zwang, Druck oder sonstigen Restriktionen im Unterschied zu früher nichts bringt und heute nur eine Verweigerungshaltung fördern würde. Weil Verantwortliche in der Jugendpastoral dies wissen, verzichten sie auf restriktive Formen und versuchen Jugendliche auf positive Weise für den christlichen Glauben zu gewinnen.

Und wie stehen über die katholisch Getauften hinaus alle bundesdeutschen Jugendlichen zu Kirche und Glauben? Zur Beantwortung dieser Frage ist auf zwei empirische Untersuchungen besonders zu verweisen: Als erstes ist die Sinus-Milieustudie zu nennen. Sie ist benannt nach dem sinus-Forschungsinstitut für Markt- und Sozialforschung mit Sitz in Heidelberg. Dieses Institut hat im Jahr 2007 eine erste Jugendstudie veröffentlicht, in der noch drei Altersgruppen unterschieden wurden: Kinder von 9 bis 13 Jahren, Jugendliche von 14 bis 19 Jahren und junge Erwachsene von 20 bis 27 Jahren. Im April 2016 ist eine zweite Nachfolgestudie veröffentlicht worden, die sich jedoch auf Teenager zwischen 14 und 17 Jahren beschränkt und sich dementsprechend U18 nennt. ${ }^{10}$ Sie ordnet die Jugendlichen einem von sieben Milieus zu, deren Mitglieder gemeinsame Wertorientierungen, Lebensauffassungen und Lebensweisen teilen. Bei den Mitgliedern eines Milieus - die Studie spricht bewusst von Milieuorientierung, weil sie darum weiß, dass sich bei Jugendlichen vieles noch ändert - zeigen sich Ähnlichkeiten zwischen den ethischen, ästhetischen, religiösen oder kirchlichen Auffassungen, was sich beispielsweise im Freizeitverhalten oder in der Einrichtung des Zimmers auswirkt. Aus kirchlicher Perspektive stimmt besonders nachdenklich, dass nicht die kirchenaffinen Milieus für Jugendliche gesellschaftlich tonangebend sind. Trendsetter sind vielmehr die kirchlich-distanzierten, , postmodernen" Milieus.

Die zweite aufschlussreiche Untersuchung zur Situation Jugendlicher in Deutschland ist die Shell-Jugendstudie. Hierbei handelt es sich um eine Langzeitstudie, welche die Lebensbedingungen, Einstellungen und Orientierungen der jungen Generation im Alter von 12 bis 25 Jahren auf

10 Ausführlich hierzu M. Calmbach u.a., Wie ticken Jugendliche 2016? Lebenswelten von Jugendlichen im Alter von 14 bis 17 Jahren in Deutschland, Heidelberg 2016. 
dem Hintergrund des sozialen Wandels exploriert. Sie wird gesponsert vom gleichnamigen Mineralölkonzern, der damit (sicher nicht ganz uneigennützig) signalisieren möchte, dass er zur Übernahme gesellschaftlicher Verantwortung bereit ist. Die erste Shell-Jugendstudie erschien im Jahr 1953, an die alle 4 bis 5 Jahre eine Nachfolgeuntersuchung mit unterschiedlichen Schwerpunkten anknüpfen konnte.

Letztes Jahr wurde die mit Spannung erwartete 17. Shell-Jugendstudie veröffentlicht. ${ }^{11}$ Sie wurde vom Münchener Forschungsinstitut TNS im Auftrag von Shell erstellt und bietet eine repräsentative, persönlichmündliche Querschnittserhebung, die durch leitfadengestützte Interviews qualitativ vertieft worden ist. Aufgrund der vorliegenden Ergebnisse charakterisiert Klaus Hurrelmann, einer der für die Studie Verantwortlichen, die Jugend Deutschlands als "Generation R wie relaxed ${ }^{\text {" }}{ }^{12}$. Damit ist gemeint: Während die Gesamtlage in den beiden letzten Jahrzehnten pessimistisch eingeschätzt wurde, blicken jetzt 52\% der Jugendlichen aufgrund der guten wirtschaftlichen Lage optimistisch in die Zukunft; $61 \%$ gehen davon aus, dass sie ihre Zukunft meistern werden ${ }^{13}$ Bemerkenswerterweise hat ihre Aufmerksamkeit für politische Themen und Zusammenhänge zugenommen: Interessierten sich 2002 nur 30\% hierfür, so sind es derzeit $41 \%$. Aufhorchen lässt indes ihre Unzufriedenheit mit dem etablierten politischen System, das vielen zu apparatehaft und zu wenig transparent erscheint. ${ }^{14} \mathrm{Neu}$ ist eine positive Identifikation mit dem eigenen Land. 62\% geben an, dass sie stolz darauf sind, Deutsche zu sein; sie sagen dies ohne einen nationalistischen Unterton und verbinden ihre Aussage mit dem Wunsch, dass das Land international eingebettet ist. ${ }^{15}$

Wie denken der Shell-Studie zufolge heutige Jugendliche über Glaube und Religion? ${ }^{16}$ Religion steht nicht im Zentrum des Wertesystems

\footnotetext{
11 Vgl. Jugend 2015. 17. Shell Jugendstudie, Hg. Shell-Deutschland, Wiesbaden 2015.

12 So in einem Interview mit der Wochenzeitschrift DIE ZEIT in der Ausgabe vom 15.10.2015, im Internet einsehbar unter http://www.zeit.de/2015/42/shell-studie-jugend-generation (abgerufen am 28.4.2016).

13 Vgl. Jugend 2015, Hg. Shell-Deutschland (s. Fußnote 10), S. 100 u. 103.

14 Vgl. Jugend 2015, Hg. Shell-Deutschland, op. cit., S. 156-159.

15 Vgl.Jugend 2015, Hg. Shell-Deutschland, op.cit., S. $227 f$.

16 Vgl. zu Folgendem ebd., S. 248-260. - Für die Jugendpastoral ist außerdem aufschlussreich, welches Bild sich heutige Jugendliche von Familie und Beruf machen und wie sie sozi-
} 
Jugendlicher, ist für sie aber gleichwohl eine konstante Größe, die zur Gesellschaft dazugehört. Für 39\% der befragten Katholiken und für 32\% der evangelischen Christen hat der Glaube eine Bedeutung für ihre Lebensführung; anno 2002 waren es noch 51\% der Katholiken und damit $12 \%$ mehr; die Bindekraft des christlichen Glaubens ist somit in der nachfolgenden Generation gesunken. Anders sieht es bei den muslimischen Jugendlichen aus; 76\% von ihnen ist der Glaube an Gott für ihr Leben wichtig. Ähnlich hoch ist die Zahl mit 68\% aber auch bei christlich-orthodoxen Jugendlichen. Freilich sind diese Gruppen gesellschaftlich nicht meinungsbildend, denn die Zahl der konfessionslosen Jugendlichen ist doppelt so hoch wie die der islamischen und orthodoxen Jugendlichen zusammen. Aufschlussreich ist die Frage nach dem Gottesbild: 29\% aller Befragten und damit nicht einmal jeder dritte Jugendliche glaubt an einen persönlichen Gott; selbst bei den getauften Jugendlichen ist es nur knapp jeder Zweite. 17\% aller Jugendlichen bekennen sich zu einer überirdischen Macht. 26\% glauben weder an einen persönlichen Gott noch an eine überirdische Macht, und 23\% wissen nicht richtig, woran sie glauben sollen. Und schließlich: Nur 15\% der evangelischen und 20\% der katholischen Jugendlichen (und damit jeder Fünfte) beten regelmäßig.

\section{Standortbestimmung: Jugendpastoral in Deutschland}

Oben wurde erwähnt, dass die gegenwärtige Erstkommunion- und Firmvorbereitung bei manchen einen faden Nachgeschmack hinterlässt. Sie wird mit sehr hohem Aufwand betrieben, ohne dass sich dies im Gottesdienstbesuch signifikant niederschlägt. Dies könnte zu der Annahme verführen, dass die ganze Vorbereitung im Grunde sinnlos und vertane Zeit sei. Dem widerspricht jedoch eine jüngst erschienene Panelstudie zur Erstkommunionkatechese, bei der die Beteiligten unmittelbar vor Beginn und nach Ende der Katechese sowie ein Jahr später befragt wurden. Im Blick der Forschung standen katholische Kinder, die

ale Netzwerke und Medien nutzen. Beiden Themenkomplexen widmet sich je ein weiteres Kapitel der Studie. 
im Jahr 2011 zur Erstkommunion geführt worden waren, ihre Eltern sowie die zuständigen Pfarrer und Pastoral- oder Gemeindereferent(inn)en; als Kontrollgruppe wurde zeitgleich eine Gruppe nicht-katholischer Kinder interviewt. ${ }^{17}$ Ein positives Resultat der Studie ist, dass die Teilnahme an der Erstkommunionkatechese sowohl die kognitive, die affektive als auch die handlungsorientierte Seite der Religiosität positiv beeinflusst, so dass die Kinder nach Abschluss des Kurses über mehr Glaubenswissen verfügen und sich auch längerfristig stärker mit Glaube und Kirche und deren Wertesystem identifizieren, als dies bei der Kontrollgruppe der nicht-katholischen Kinder der Fall ist. Auf die Religiosität der Eltern und ihre Einstellung zur Kirche wirkt sich die Teilnahme der Kinder an der Erstkommunionkatechese ebenfalls positiv aus, wenn auch etwas schwächer. Diese Studie bietet eine Fülle von Einzelerkenntnissen, die längst nicht alle ausgewertet sind. Es wäre wünschenswert, eine vergleichbare Studie auch im Blick auf die jugendlichen Firmanden in Angriff zu nehmen, was bisher leider ein Desiderat ist. ${ }^{18}$

Was sind über die Sakramentenpastoral hinaus wichtige Orte der Jugendpastoral in Deutschland? Die breite Palette an Angeboten lässt sich nicht vollständig aufzählen, nur einige markante Beispiele seien genannt. Nach wie vor treffen und engagieren sich Jugendliche in der Pfarrei. Vor allem die Messdiener haben sich in den letzten Jahrzehnten zu einem Erfolgsmodell entwickelt, um die so mancher evangelische Pfarrer seine katholischen Kollegen beneidet; über den liturgischen Dienst hinaus sind die Messdiener in vielen Gemeinden eine lebendige Gemeinschaft. Einige Jugendgruppen in der Pfarrei sind verbandlich organisiert und damit an überpfarrliche Strukturen rückgebunden, etwa die Katholische Junge Gemeinde (KJG), die Pfadfinder (DPSG) oder die Kolpingjugend. Sie sind ihrerseits in der Dachorganisation des BDKJ (Bunds der Deutschen Katholischen Jugend) zusammengefasst, der als Koordinationsgremium und eine Art gesellschaftlicher Interessenvertretung katholischer

17 Vgl. ausführlich hierzu Forschungsgruppe „Religion und Gesellschaft“: Werte - ReligionGlaubenskommunikation. Eine Evaluationsstudie zur Erstkommunionkatechese, Wiesbaden 2015.

18 Zur Firmpastoral vgl. Ph. Müller, Praxisfeld: Firmvorbereitung. Jugendlichen Firmanden in einer pluralen Gesellschaft den Glauben anbieten, [in]: Relativismus. Der Anspruch des christlichen Glaubens in einer pluralen Gesellschaft, Hg. Ph. Müller, W. Müller-Geib, München 2010, S. 73-95. 
Jugendlicher fungiert. Zwei besondere Aktionen des BDKJ seien eigens erwähnt:

- Die Sternsinger: In Verbindung mit dem Kindermissionswerk ziehen in der Zeit um Epiphanie die als die Heiligen Drei Könige gekleideten Kinder von Haus zu Haus, um den Segen Gottes zu bringen und für notleidende Kinder anderer Länder und Kontinente zu sammeln. Im Jahr 2014 haben 330.000 Kinder und Jugendliche 78,41 Millionen Euro gesammelt. ${ }^{19}$

- Die 72-Stunden-Aktion: Hier setzen katholische Jugendgruppen innerhalb von 72 Stunden ein soziales, interkulturelles oder ökologisches Projekt um und rücken Menschen wie Behinderte, Arbeitslose oder alte und einsame Menschen in den Blick, die sonst schnell vergessen werden. Bei der letzten 72-Stunden-Aktion im Juni 2013 haben über 4.000 Projektgruppen mit über 150.000 Teilnehmern ihr Projekt in die Tat umgesetzt. Ein großer, vor allem bei jungen Menschen beliebter Radiosender hat diese Aktion medienwirksam begleitet.

In einigen Diözesen (und zwar häufig in Städten) ist in den letzten Jahren die eine oder andere „klassische“ Pfarrkirche zu einer Jugendkirche umgewandelt geworden mit speziellen pastoralen und liturgischen Angeboten für Jugendliche. Auch die Orden, beispielhaft seien die Salesianer Don Boscos erwähnt, und die Neuen Geistlichen Bewegungen (wie z. B. Schönstatt) leisten einen originären jugendpastoralen Beitrag. Vielen ist Taizé im französischen Burgund ein Begriff; nicht wenige Jugendliche, die eine Woche dort waren, sind dort zu den Quellen des christlichen Glaubens vorgestoßen und haben dort einen Sinn für ihr Leben entdeckt. Natürlich darf auch der Weltjugendtag nicht unerwähnt bleiben, der im Jahr 2005 in Köln stattfand. Aus der Vigilfeier dieses Weltjugendtags ist "Nightfever“ entstanden, das sich zu einem weltweiten "Exportschlager" entwickelt hat; bei „Nightfever" handelt es sich um offene Gebetsabende im Dienst der Neuevangelisierung, in deren Mitte die eucharistische Anbetung steht. Ein Grund dafür, weshalb Formate wie Jugendkirchen, der Weltjugendtag oder auch "Nightfever" heutige Jugendliche so ansprechen, hat damit zu tun, dass im Rahmen heutiger Erlebnisorientierung Events eine wichti-

19 Katholische Kirche in Deutschland: Zahlen und Fakten 2014/15, op. cit., (s. Fußnote 3), S. 52 f. 
ge Rolle spielen. Events mit ihren spezifischen ästhetischen und kulturellen Ausdrucksformen haben den Charakter des Außergewöhnlichen; sie durchbrechen den geregelten Alltag, vermitteln ein Gefühl temporärer Zugehörigkeit, ohne vom einzelnen eine dauerhafte und feste Entschiedenheit einzufordern. ${ }^{20}$

An welchen theologischen Kriterien richtet sich die Jugendpastoral in Deutschland aus? Welche lehramtlichen Dokumente sind relevant? Bedeutsam sind neben den Texten des Zweiten Vatikanischen Konzils der Synodenbeschluss aus dem Jahr 1975 sowie die "Pastoralen Leitlinien“ aus dem Jahr 1991. Zunächst zum Synodenbeschluss: Um nach Wegen zu suchen, wie die Ergebnisse des Zweiten Vatikanischen Konzils in der deutschen Kirche rezipiert werden können, haben die Bistümer des Landes von 1971-1975 im Würzburger Dom eine Synode durchgeführt, die als „Würzburger Synode“ bezeichnet wird. Das Ergebnis sind 18 Beschlüsse und 6 Arbeitspapiere, darunter auch der Synodenbeschluss „Ziele und Aufgaben kirchlicher Jugendarbeit" ${ }^{21}$ Adressaten dieses Beschlusses sind die Verantwortlichen der Jugendpastoral, aber auch die kirchennahen Jugendlichen selbst als „die ersten und nächsten Zeugen des christlichen Glaubens, die ersten und nächsten Vertreter der Kirche“22. Wie die Pastoralkonstitution Gaudium et spes bei der Lebenswelt der Menschen und den „Zeichen der Zeit" ihren Ausgangspunkt nimmt, so geht auch dieser Text induktiv vor und setzt nach einer Einleitung (1.) mit einer Analyse der gesellschaftlichen und psycho-sozialen Bedingungen Jugendlicher ein (2.); hieran schließen sich anthropologische und theologische Aspekte an (3.), bevor das Dokument auf das Angebot (4.) und die Erfordernisse (5.) kirchlicher Jugendarbeit zu sprechen kommt. Der Text schließt mit jugendpolitischen Forderungen (6.). Das Dokument bringt zum Ausdruck: Die Kirche will jungen Menschen selbstlos dabei helfen, das zu finden, wonach sie sich zutiefst sehnen, nämlich Glück,

20 Über Events als Phänomen der Gegenwartskultur vgl. Hobelsberger, Jugendpastoral des Engagements (s. Fußnote 8), S. 327-334.

${ }_{21}$ Der Text ist abgedruckt in: Gemeinsame Synode der Bistümer in der Bundesrepublik Deutschland. Beschlüsse der Vollversammlung (Offizielle Gesamtausgabe), Freiburg. Br. 2012, S. 288-311.

22 Gemeinsame Synode der Bistümer..., op.cit., S. 289. 
Liebe, Friede, Freude und Heil, und sie ist gleichzeitig zutiefst überzeugt, dass all dies in Jesus Christus gefunden werden kann. ${ }^{23}$ Dem „personalen Angebot" kommt ein eindeutiger Vorrang vor dem "Sachangebot" zu: zunächst durch die Gruppe der gleichaltrigen Jugendlichen selbst, denen Begegnungs- und Erfahrungsräume zur Verfügung zu stellen sind, aber auch durch die erwachsenen Begleiter, die sich als Glaubenszeugen auf die Fragen der Jugendlichen einlassen..$^{24}$ Im Unterschied zu einem bis dahin dominanten kerygmatischen Konzept von Jugendpastoral, das die Verkündigung von Glaubensinhalten in den Mittelpunkt stellt, ist der Würzburger Synodenbeschluss stark diakonisch ausgerichtet, wenn er christliche Jugendarbeit als ,selbstlose[n] Dienst an den jungen Menschen und an der Gestaltung einer Gesellschaft “25 versteht.

Bis heute gilt der Synodenbeschluss als ein Meilenstein der Jugendpastoral in Deutschland.

GleichwohlwurdeschonfrüheinMangel ankonkretenHandlungsprinzipien und-konzepten sowie eine mangelnde theologische Fundierung beklagt. ${ }^{26}$ Dieses Defizit suchten die „Leitlinien zur Jugendpastoral“ zu kompensieren, welche die Pastoralkommission der Deutschen Bischofskonferenz ein Jahr nach der Wiedervereinigung Deutschlands im Jahr 1991 verabschiedet hat. ${ }^{27}$ Die Leitlinien machen die Absichten und den Auftrag der Jugendpastoral transparent (1.), gehen aber auch auf die Lebenswirklichkeit Jugendlicher ein (2.). Die Ziele der Jugendpastoral (3.) werden durch den Beziehungs-Begriff trinitarisch begründet: Wie der dreieine Gott in sich lebendige Beziehung ist, so verwirklicht auch der Mensch als Abbild Gottes sein Leben wesentlich in Beziehungen: zu Gott, zum Nächsten, zur Schöpfung und zu sich selbst. Aus diesem Axiom der theologischen Anthropologie werden an- und abschließend Gestaltungselemente (4.)

\footnotetext{
23 Vgl. Gemeinsame Synode der Bistümer..., op. cit., S. $294 \mathrm{f}$.

24 Vgl. Gemeinsame Synode der Bistümer..., op. cit., S. 298-302.

25 Gemeinsame Synode der Bistümer..., op. cit., S. 294.

26 Vgl. G. Biemer, Der Dienst der Kirche an der Jugend. Grundlegung und Praxisorientierung, [in:] Handbuch kirchlicher Jugendarbeit (Bd. 1), Freiburg i. Br. 1985, S. 150-152.

27 Pastoral-Kommission der deutschen Bischöfe, Leitlinien zurJugendpastoral, 20. September 1991, Bonn ohne Jahr. Man beachte den im Vergleich zur Würzburger Synode veränderten Sprachgebrauch von „kirchlicher Jugendarbeit“ zu "Jugendpastoral“.
} 


\section{Philipp Müller}

und Strukturen (5.) der Jugendpastoral abgeleitet. Insgesamt wollen die "Jugendpastoralen Leitlinien" nur einen Rahmen vorgeben. Die einzelnen Bistümer werden ermuntert, auf dieser Grundlage diözesane Leitlinien zu entwickeln, die zu den konkreten Gegebenheiten vor Ort passen; einige Bistümer sind dieser Anregung nachgekommen.

Die ,Jugendpastoralen Leitlinien“"wurden trotz ihrer soliden theologischen Fundierung längst nicht in derselben Weise wie der Synodenbeschluss rezipiert. Mittlerweile sind sie schon ein Vierteljahrhundert alt, und es wäre von bischöflicher Seite an der Zeit, erneut ein Grundlagendokument zu erarbeiten, das die Situation der jetzigen Jugend in Deutschland zum theologisch-pastoralen Grundauftrag von Kirche in Beziehung setzt.

\section{Ein Bonmot Klaus Hemmerles}

Im Jahr 1983 hat Bischof Klaus Hemmerle Fragen gestellt, die heute ebenso aktuell sind wie damals: „Was fängt die Jugend mit der Kirche an? Was fängt die Kirche mit der Jugend an?" In seinen Überlegungen geht er der Frage nach, ob es für die Kirche wichtiger sei, sich ganz auf die Jugendlichen einzulassen, und dabei Gefahr zu laufen, die Botschaft zu verkürzen, oder die Botschaft des Evangeliums zu verkünden und dabei vielleicht die Jugendlichen in ihrer Lebenswelt nicht richtig zu verstehen. Hemmerle lässt sich in seinen Überlegungen nicht auf ein „entweder - oder“ ein, denn für ihn gehört beides zuinnerst zusammen: Sich den heutigen Jugendlichen zuzuwenden, sie anzunehmen und zu verstehen suchen ist der Weg, durch den das Evangelium zu den Menschen kommen kann. Mehr noch: Wer sich auf die Jugendlichen einlässt und ebenso im Evangelium verwurzelt ist, dem werden Aspekte des christlichen Glaubens aufscheinen, die ihm bisher noch nicht in derselben Weise bewusst waren. So kann Hemmerle formulieren: „Lass mich dich lernen, dein Denken und Sprechen, dein Fragen und Dasein, damit ich daran die Botschaft neu lernen kann, die ich dir zu überliefern habe." ${ }^{28}$

${ }_{28}$ K. Hemmerle, Was fängt die Jugend mit der Kirche an? Was fängt die Kirche mit der Jugend an?, op. cit. (s. Fußnote 1), S. 329. 
Freilich weisen die oben genannten aktuellen Jugendstudien darauf hin, dass es in der pastoralen Praxis ein sehr anspruchsvolles Unterfangen ist, sich Jugendlichen unterschiedlicher Milieus zuzuwenden, die weitgehend anderen Wertorientierungen folgen und die einen Lebensstil pflegen, der einem selbst bisweilen befremdlich vorkommen mag. Auch Klaus Hemmerle war sich der Herausforderungen bewusst, die auf einen Jugendseelsorger zukommen können. Hiervon zeugt die folgende Anekdote: Als einer seiner bischöflichen Mitbrüder zum Jugendbischof ernannt wurde, ging er auf ihn zu und sagte zu ihm: „Ich gratuliere Dir zu Deiner neuen Aufgabe und möchte Dir drei gute Ratschläge mit auf den Weg geben. Erstens: aushalten. Zweitens: aushalten. Drittens: aushalten." Hemmerle wollte damit sagen: Weil Jugendliche eigene Denk- und Lebensweisen haben und kirchliche Positionen kritisch zur Disposition stellen, kann die Jugendpastoral für den Jugendseelsorger eine echte Herausforderung und manchmal auch eine Zumutung sein. Freilich hat sich die Kirche durch das Zweite Vatikanische Konzil selbst dazu verpflichtet, diesen Weg zu gehen, sind doch gemäß der Eröffnungspassage von Gaudium et spes „Freude und Hoffnung, Trauer und Angst der Menschen von heute ... auch Freude und Hoffnung, Trauer und Angst der Jünger Christi. Und es gibt nichts wahrhaft Menschliches, das nicht in ihren Herzen seinen Widerhall fände. “ ${ }^{29}$

Bereits gut eine Generation vor dem Zweiten Vatikanischen Konzil hat der Schweizer Psychiater Carl Gustav Jung vor elsässischen Seelsorgern das schöne Wort geprägt: „Man wandelt nur das, was man annimmt.“30 Was individualpsychologisch gilt, trifft ebenso auf die Jugendpastoral zu: Nur wenn man Jugendliche zunächst einmal annimmt, wie sie sind und eine Beziehung zu ihnen aufbaut, kann sich auch ein Weg weisen, ihnen die Botschaft des Evangeliums nahezubringen. Wer sich in dieser Weise auf Jugendliche einlässt, wird vielleicht selbst der Beschenkte sein: durch das Vertrauen, das ihm Jugendliche entgegenbringen, aber auch

29 Gaudium et spes, 1.

30 Dies war auf der elsässischen Pastoralkonferenz zu Straßburg im Mai 1932. Vgl. C. G. Jung, Zur Psychologie östlicher und westlicher Religion, [in:] Gesammelte Werke (Bd. 11), 2. Aufl., Olten 1973, S. 367. 
Philipp Müller

durch einen neuen und frischen Blick auf das Evangelium, der sich aus der Begegnung mit den Jugendlichen ergibt. 


\section{Summary}

\section{Jugend und Kirche in Deutschland - Eine pastoraltheologische Skizze}

Dieser Beitrag stellt zunächst dar, was heutige Jugendliche in Deutschland, von denen weniger als ein Drittel katholisch getauft ist, über Glaube und Kirche denken. Anschließend kommt die breite Praxis der Jugendpastoral in Deutschland zur Sprache, aber auch theoretische Konzepte, denen sie sich verpflichtet weiß. Der Beitrag schließt mit einem Plädoyer, sich als Seelsorger auf die Jugendlichen von heute und ihre existentiellen Fragen wirklich einzulassen.

Schlüsselwörter: Jugendpastoral, Deutschland, Sakramente, Religionsunterricht, Jugendsoziologie

\section{The Youth and Church in Germany - A Pastoral-Theological Outline}

This article at first describes what today's young German people - less than a third of whom are baptized catholic - think about believe and about the Church. Then the diversified practice of youth pastoral care is raised, but also the theoretical concepts on which this practice is predicated. The article finishes with a plea that persons in pastoral care should really get involved with the present-day adolescents and their existential questions.

Keywords: youth ministry, Germany, sacraments, religious education, sociological studies of youth

\section{Bibliographie}

Biemer G., Der Dienst der Kirche an der Jugend. Grundlegung und Praxisorientierung, [in:] Handbuch kirchlicher Jugendarbeit (Bd. 1) Freiburg i. Br. 1985.

Calmbach M. u.a., Wie ticken Jugendliche 2016? Lebenswelten von Jugendlichen im Alter von 14 bis 17 Jahren in Deutschland, Heidelberg 2016.

Forschungsgruppe „Religion und Gesellschaft", Werte - Religion-Glaubenskommunikation. Eine Evaluationsstudie zur Erstkommunionkatechese, Wiesbaden 2015.

Gemeinsame Synode der Bistümer in der Bundesrepublik Deutschland. Beschlüsse der Vollversammlung (Offizielle Gesamtausgabe), Freiburg i. Br. 2012.

Hemmerle K., Spielräume Gottes und der Menschen. Beiträge zu Ansatz und Feldern kirchlichen Handeln, [in:] Ausgewählte Schriften (Bd. 4), Freiburg-Basel-Wien 1996, S. 324-339.

Hemmerle K., Was fängt die Jugend mit der Kirche an? Was fängt die Kirche mit der Jugend an? „Internationale katholische Zeitschrift Communio“ 12 (1983), S. 306-317. 


\section{Philipp Müller}

Hobelsberger H., Jugendpastoral des Engagements. Eine praktisch-theologische Reflexion und Konzeption des sozialen Handelns Jugendlicher, [in:] Studien zur Theologie und Praxis der Seelsorge (Bd. 67), Würzburg 2006.

Jugend 2015. 17. Shell Jugendstudie, Hg. Shell-Deutschland, Wiesbaden 2015.

Jung C. G., Zur Psychologie östlicher und westlicher Religion, [in:] Gesammelte Werke (Bd. 11), 2. Aufl. Olten 1973.

Katholische Kirche in Deutschland: Zahlen und Fakten 2014/15, Hg. Sekretariat der Deutschen Bischofskonferenz (Arbeitshilfen 275).

Müller Ph., Die katholische Kirche in Deutschland - Eine Bestandsaufnahme, „Polonia Sacra" 20 (2016) Nr. 2, S. 47-69.

Müller Ph., Praxisfeld: Firmvorbereitung. Jugendlichen Firmanden in einer pluralen Gesellschaft den Glauben anbieten, [in:] Ph. Müller, W. Müller-Geib, Relativismus. Der Anspruch des christlichen Glaubens in einer pluralen Gesellschaft, München 2010, S. 73-95.

Pastoral-Kommission der deutschen Bischöfe, Leitlinien zur Jugendpastoral, 20. September 1991, Bonn ohne Jahr.

Pastoralkommission der deutschen Bischöfe, Sakramentenpastoral im Wandel. Überlegungen zur gegenwärtigen Praxis der Feier der Sakramente - am Beispiel von Taufe, Erstkommunion und Firmung (Die deutschen Bischöfe - Pastoral-Kommission 12), Juli 1993, Bonn ohne Jahr.

Prokopf A., Religiosität Jugendlicher. Eine qualitativ-empirische Untersuchung auf den Spuren korrelativer Konzeptionen, [in:] Praktische Theologie heute (Bd. 98), Stuttgart 2008.

Rödder A., 21.0. Eine kurze Geschichte der Gegenwart, 2. Aufl. München 2015.

Simon W., Religionsunterricht. Praktisch-theologisch, [in:] Lexikon für Theologie und Kirche, 3. Aufl. (Bd. 8), S. 1077-1079.

Ziebertz H. G., Kalbeim B., Riegel U., Religiöse Signaturen heute. Ein religionspädagogischer Beitrag zur empirischen Jugendforschung, [in:] Religionspädagogik in pluraler Gesellschaft, (Bd. 3), Gütersloh-Freiburg 2003. 